\title{
Linx
}

Revue des linguistes de l'université Paris X Nanterre

52 | 2005

Lexique, terminologie, discours

\section{La terminologie entre science et discours? Remarques sur la terminologie institutionnelle}

Danielle Candel

\section{(2) OpenEdition}

\section{Journals}

Édition électronique

URL : http://journals.openedition.org/linx/196

DOI : $10.4000 /$ linx. 196

ISSN : 2118-9692

Éditeur

Presses universitaires de Paris Nanterre

\section{Édition imprimée}

Date de publication : 1 juin 2005

Pagination : 85-96

ISSN : 0246-8743

\section{Référence électronique}

Danielle Candel, «La terminologie entre science et discours? Remarques sur la terminologie

institutionnelle », Linx [En ligne], 52 | 2005, mis en ligne le 27 janvier 2011, consulté le 30 avril 2019

URL : http://journals.openedition.org/linx/196 ; DOI : 10.4000/linx.196 


\title{
La terminologie entre science et discours? Remarques sur la terminologie institutionnelle
}

\author{
Danielle Candel \\ Histoire des Théories Linguistiques, UMR 7597 CNRS - \\ Université Paris 7
}

Cet intitulé est inspiré du titre de l'ouvrage de Marie-Françoise Mortureux, La lexicologie entre langue et discours, paru en 1997 chez SEDES. La simplicité de cette formule si éclairante pourrait-elle être reprise et servir à mieux situer une branche de cette autre discipline, cousine de la lexicographie, qu'est la terminologie ? Sans oser pousser trop loin ce rapprochement sans doute inattendu, il semble bien que l'on trouve chez cet auteur, spécialiste du discours de vulgarisation scientifique ${ }^{1}$, un bon guide pour la description de discours et de pratiques terminologiques.

«Sans cette relation entre la langue et les discours, entre le lexique de la langue et les vocabulaires des discours, il n'y a pas de communication ; car celle-ci s'appuie toujours sur l'adaptation à des cas particuliers d'un outil, d'un instrument commun à tous les locuteurs; cet instrument est leur langue, qu'ils utilisent en discours» (Mortureux 1997 : 5). Le processus de communication ainsi décrit est le même quel que soit l'objet de la communication. Tout en considérant la sphère terminologique, nous nous situons, de ce fait, dans la même réalité que celle expliquée par M.-F. Mortureux. Mais comment transformer pour le compte de la terminologie la déclaration faite au sujet de la lexicologie?

\footnotetext{
${ }^{1}$ V. notamment, dans cette mouvance, Jacobi 1997.
} 
La terminologie a pour objet l'étude d'un vocabulaire spécifique, relevant d'un domaine de spécialité et dont les usagers forment un groupe relativement identifiable. Cette caractéristique offre le consensus nécessaire aux échanges discursifs, voire, au développement ou à l'enrichissement lexical. Le paramètre à introduire nous semble donc être l'aspect couvert par "spécialité », « science », «savoir », «technique » ou encore «technologie», qui en sont représentatifs. Par souci de concision, nous recourons au terme «science», auquel nous conférons ici une valeur d'hyperonyme. Nous ferons le choix, pour ce qui nous occupe, de considérer le paramètre «langue » comme implicite. Nous avons donc opté pour le raccourci suivant: «la terminologie entre science et discours ».

En posant, à côté de l'ensemble formé par «lexicologie », « langue » et « discours », l'ensemble formé par «terminologie », «science» et « discours», une constante se dégage : c'est l'élément « discours ». On introduit d'autre part un parallélisme entre «lexicologie » et «terminologie», termes qui réfèrent tous deux à des disciplines et pratiques. Il y a enfin, comme on le verra, report de l'élément supprimé («langue») sur l'élément ajouté (« science ») : on charge de ce fait l'élément «science » des valeurs de «norme» et de « système », celles-là mêmes qui étaient incluses dans l'élément « langue »².

Le titre choisi par M.-F. Mortureux pourrait être ambigu : la valeur de «entre » est aussitôt précisée : la lexicologie se situe bien au croisement de la «langue » et du « discours », et n'est pas isolée de l'un ou de l'autre élément. Mais qu'en serait-il de notre formule ? La terminologie relève-t-elle bien de la «science » et du " discours », ou bien, au contraire, serait-elle d'une manière ou d'une autre exclue de l'un de ces deux pôles ? Nous réservons à plus tard la réponse à cette question.

Il importe d'abord de rappeler des définitions courantes de " terminologie » (I). Puis nous voudrions montrer la place de la terminologie à la croisée des chemins entre " science» et « discours ». Pour cela, nous présenterons les valeurs et les rôles de la "science », telles qu'ils nous apparaissent à travers l'expérience de la pratique terminologique institutionnelle (II). Nous rappellerons ensuite l'élément commun aux deux relations exprimées, c'est-à-dire le « discours », dont nous analyserons la nature et le rôle (III). Pour finir, nous nous interrogerons sur le poids de la norme prescriptive dans ce circuit de terminologie institutionnelle et sur le rôle, dans ce circuit, des véritables décideurs (IV).

\section{Une terminologie « stricte »}

La branche de la discipline que nous étudions est la terminologie institutionnelle. Dans la pratique, elle est censée produire, domaine par domaine, des listes de vocabulaire. Elle correspond aux pratiques mises en place dans le cadre du processus d'enrichissement de la langue française et nous la nommons volontiers « terminologie ponctuelle multipôle». Elle est «ponctuelle» et non systématique, elle n'implique pas de faire le tour d'un domaine pour traiter systématiquement l'ensemble

\footnotetext{
${ }^{2}$ La définition suivante de « langue générale » dans Boutin-Quesnel et al. (1985) : «Partie du système linguistique comprise et utilisée par la majorité des locuteurs d'une communauté linguistique» renvoie au système.
} 
des termes d'un même sous-ensemble. Nous avons par ailleurs souhaité introduire le terme «multipôle» pour insister sur la pluralité et la variété des auteurs, acteurs et récepteurs impliqués dans le processus officiel d'enrichissement de la langue française. Nous nous sommes expliquée ailleurs sur cette appellation et avons aussi exposé cette activité dans Candel 1999, 2002 et 2003. Y participent des experts du domaine; nous y sommes en tant qu'expert linguiste.

La terminologie est couramment présentée en opposition avec la lexicologie. Elle est considérée comme éloignée des réalisations discursives. Les "termes » sont caractérisés par un lien sans équivoque avec le domaine précis dont ils relèvent et, surtout, par la relation de biunivocité qu'ils entretiennent avec le concept. Ces principes sont décrits et/ou remis en cause par nombre d'auteurs (Cabré 1998, Pearson 1998, Humbley 2001, Aussenac-Gilles et Condamines 2003). J. Humbley (2004) établit de son côté l'historique d'un ensemble de mouvements opposés à une telle conception rigoureuse de la terminologie, retracé à partir d'un riche corpus d'articles critiques.

Cette attitude critique émerge notamment de la lecture du Dictionnaire de linguistique. Dubois et al. 1999 rappellent que la terminologie est d'abord caractérisée par l'étude systématique des concepts: «On appelle également terminologie l'étude systématique de la dénomination des notions (ou concepts) spécifiques de domaines spécialisés des connaissances ou des techniques (...)» (article «terminologie » : 481). En vérité, l'article «terminologie» semble presque avoir été introduit dans cette seconde édition du dictionnaire pour servir de tremplin à l'article «socioterminologie ». En effet, la terminologie réductrice s'oppose nettement à cette autre science du langage, notamment en négligeant totalement les particularités langagières des différents groupes de locuteurs : «Pour se démarquer de cette école indifférente aux aspects sociolinguistiques, des terminologues francophones ont avancé, depuis les années 80, la notion de socioterminologie » (article «terminologie »: 481).

L'article socioterminologie permet d'ailleurs un rappel de ces réalités défectueuses de la terminologie : «La socioterminologie veut prendre en compte les aspects sociolinguistiques de la communication scientifique et technique. Elle préfère la notion de sphère d'activité à celle du domaine, en considération des nouveaux rapports qu'entretiennent sciences, technique et production (...). La socioterminologie refuse de donner priorité à la notion dans l'étude des vocabulaires spécialisés, s'opposant ainsi à la terminologie majoritaire inspirée d'E. Wüster» (article "socioterminologie » : 436).

Enfin, la mise en avant de ces limitations imposées par la théorie terminologique ressort aussi, très largement, de l'article «terme »: «En terminologie, le terme ou unité terminologique est l'unité signifiante constituée d'un mot (terme simple) ou de plusieurs mots (terme complexe), qui désigne une notion de façon univoque à l'intérieur d'un domaine (définition de l'Office de la langue française du Québec) (article «terme» : 480). C'est en somme ce qu'exprime Mortureux (1997b : 111) : «termes, mots strictement définis dans les discours scientifiques et techniques ». Mais le dictionnaire de Dubois précise: «Cette définition, fidèle à l'enseignement de E. Wüster, fait bien ressortir la limitation du terme à la face signifiante du signe linguistique. Mais il ne faut pas confondre pour autant le terme ainsi conçu avec le 
signifiant saussurien (v. système notionnel). Loin qu'on ait union du signifiant et du signifié sous l'effet du système, on a, dans cette conception, des notions (issues d'aspects du référent) qui s'organisent en systèmes : le terme n'est alors, comme on le voit dans la définition, que la traduction linguistique univoque d'une notion qui lui préexiste » (article « terme »: 480).

Le dictionnaire de Dubois va donc dans le sens de la critique bien établie de cette terminologie que l'on pourrait appeler «traditionnelle». Cependant, il semble qu'il y ait lieu de moduler et de corriger cette vue de la terminologie «stricte », celle que l'on appelle aussi la «terminologie majoritaire» (Humbley 2001 et 2004). Étudier le mouvement des idées en terminologie entraîne à analyser par exemple la représentation que l'on se fait ou que l'on a pu se faire, dans certains courants ou à certaines époques, du «terme» par rapport au «mot». Et il peut y avoir amalgame entre des prises de position en «terminologie de la terminologie» et des pratiques terminologiques effectives. Dans les travaux des Commissions de terminologie, en particulier, quelle est la situation? Est-il possible de dire dans quelle mesure on s'attache à décrire les concepts scientifiques en eux-mêmes, tels que connus par les spécialistes, par les experts des domaines eux-mêmes, plutôt qu'à décrire le fonctionnement et l'actualisation en langue de leurs dénominations et de leurs usages?

\section{Rôles et valeurs de « la science » en terminologie}

Rappelons une nouvelle fois le parallélisme que nous tentons avec la formule de M.-F. Mortureux. Le pôle de la « science » y représente le système, la norme ; c'est en somme un pôle de stabilité, qui rassemble les locuteurs, et qui sert de référence pour évaluer leurs parlers dans leurs variétés, tels que perceptibles à travers leurs réalisations discursives. Une telle fonction de stabilité liée au paramètre «langue » est aussi assurée par le paramètre «science », celui-là même qui, comme nous le signalions plus haut, le supplante en partie dans le cadre de cette étude.

Nous souhaitons surtout insister sur le rôle important de ce paramètre «science ». Il émerge clairement lorsque nous nous situons dans la perspective de la pratique des commissions spécialisées de terminologie et de néologie. Nous entendons aussi bien, par «science», la technique, les sciences et leurs applications, bref, l'expertise spécialisée : c'est avant tout le rôle de l'expert qui s'impose ici. C'est lui qui tranche, qui concourt à sélectionner les dénominations et à forger les définitions exactes. La compétence est celle de l'expert du domaine. Les spécialistes sont en somme amenés à se reformuler, à transférer eux-mêmes, et très directement, leur propre savoir ${ }^{3}$, à l'occasion de ce travail terminologique et définitoire. Le savoir scientifique, la connaissance du domaine ou de la pratique spécialisés servent de référence. Le rôle de la science et du scientifique, des sciences et des scientifiques, techniciens, ingénieurs, experts, est central.

\footnotetext{
${ }^{3}$ Voir, sur ces questions en général, les travaux de M.-F. Mortureux et aussi Jacobi 1987.
} 


\section{Quelques exemples du recours indispensable au savoir expert}

Quelle décision prendre à partir d'un ensemble inhomogène de données recueillies? Les sources utilisées ne prêtent pas toujours au consensus - si d'ailleurs c'était le cas, les nouvelles fiches terminologiques seraient sans doute inutiles. Les deux exemples suivants ont trait à la définition, le troisième, au champ « note ».

\section{Premier exemple (la définition)}

Dans le domaine des Sciences et techniques spatiales, quel terme exact utiliser en français pour rendre chugging et chuffing, et de quelles définitions accompagner ces termes (Candel et Tolédano 2001) ? Les termes français retenus, déjà bien en usage dans le domaine, sont ronflement et halètement. L'expert linguiste, le généraliste, se sent d'abord relativement à l'aise avec de tels termes, qui ne lui sont pas étrangers, puisqu'il les rencontre aussi dans la langue générale. Il pense les connaitre. Leur traitement occasionne pourtant d'importantes difficultés d'évaluation pour ce non-spécialiste, non familier de leurs usages spécialisés. Comme ces termes sont déjà bien en usage, en anglais comme en français, les dictionnaires sont éloquents, et donc d'une grande utilité. Or on note ici une difficulté courante en lexicographie générale.

Partons du terme anglais, ce qui est la démarche habituelle en Commission de terminologie. Le Academic Press Dictionary for science and technology donne pour chugging [ronflement $]^{4}$, un sens $1:$ :In a liquid rocket engine or other aircraft engine, a combustion instability [instabilité de combustion] characterized by pulsing operation and noise at low frequencies » et un second sens, métonymie du premier : 2. "The noise [bruit] made in this type of combustion ». La recherche est faite également à partir du terme français. À lire les dictionnaires, on constate que le définisseur (ou incluant) peut être soit "une caractéristique», soit "un bruit», soit encore "une instabilité de combustion». Le terme ronflement [chugging] est défini comme une « instabilité de combustion $»^{5}$ dans des définitions en français (GDT, Termium, Mots de la combustion 1993, Dictionnaire compact 1990) et en anglais (dans Termium, Williamson 1990, Academic Press dictionary 1992), et comme un "bruit" dans une définition anglaise (Academic Press Dictionary 1992). Le terme balètement et son équivalent anglais chuffing sont, quant à eux, définis comme une «instabilité de combustion » dans quatre sources françaises (GDT, Mots de la combustion 1993, Gourseau F-A 1998, Dictionnaire compact 1990 ) et comme un «bruit» dans une définition en français et dans une autre en anglais (Termium). L'expérience en Commission de terminologie montre que, pour les spécialistes du domaine, pas plus que pour ces lexicographes, il n'est simple de décider du meilleur mode de définition. En effet, trois possibilités demeurent au sujet de ces deux éléments de sens ("instabilité de combustion» ou sa métonymie, «bruit») : un traitement équivalent pour chacun d'eux, un traitement mettant davantage en valeur l'un que l'autre, ou encore l'occultation de l'un des deux au profit de l'autre. Une

\footnotetext{
${ }^{4}$ C'est nous qui traduisons.

${ }^{5} \mathrm{Ou}$ une « forme d'instabilité de combustion ».
} 
conclusion s'impose en tout cas : sans les spécialistes, on ne pourrait, en toute conscience, définir, aussi éclairants les dictionnaires d'un côté, les contextes discursifs de l'autre, soient-ils.

\section{Deuxième exemple (la définition)}

Quelle définition proposer pour la fiche surbaissement d'orbite? Une proposition de définition de la même commission spécialisée dans le vocabulaire spatial, avait été : «Modification d'orbite consistant à diminuer l'altitude moyenne d'un satellite artificiel », puis une définition «Abaissement d'orbite destiné notamment à avancer la retombée du satellite ou à accomplir une observation rapprochée de la surface du sol » et finalement la définition «Manœuvre consistant à faire passer un satellite artificiel de son orbite initiale à une orbite d'altitude notablement inférieure, en vue de l'accomplissement d'un aspect particulier de sa mission». Seule solution pour le traitement de telles fiches : le consensus doit être recherché au sein du groupe des spécialistes - un non-spécialiste ne peut pas grand chose dans ce travail ${ }^{6}$.

\section{Troisième série d'exemples (la note)}

L'analyse du champ «note» met en lumière nombre d'indications spécialisées qui relèvent directement du savoir expert. "Note» est défini dans Boutin-Quesnel $e t$ al. (1985) ainsi : «Énoncé qui donne des informations de nature encyclopédique ou notionnelle, linguistique (flexion, mise en discours, etc.) ou terminologique (rapports terme-notion, rapports entre notions, marques d'usage, etc.) sur le terme étudié $»^{7}$.

Les listes de l'informatique et de la chimie parues respectivement aux J.0. du 27 février et 8 octobre 2003 offrent de nombreuses notes, qui se surajoutent donc aux définitions. Plusieurs d'entre elles sont de nature clairement linguistique, voire métalinguistique. Ce genre d'intervention a trait le plus souvent à des précisions données dans le but de fournir explicitement une «motivation» au signe introduit. Ainsi par exemple : «Forme elliptique de (...)» (sous orbitale); «De l'allemand Zwitter (...) » (sous zwitterion). Il peut alors arriver que cette donnée linguistique soit d'abord, et bien plus nettement, une précision conceptuelle de la part du spécialiste: «Les structures limites ont été désignées dans certains cas par l'expression «forme canonique» (sous structure limite) ou: "Traditionnellement utilisé pour qualifier les hydrocarbures dont la formule comporte un ou plusieurs noyaux benzéniques, ce terme est parfois employé comme substantif pour les désigner» (sous aromatique). Il semble que, prétextant une précision d'ordre linguistique, le spécialiste en profite

\footnotetext{
${ }^{6}$ Une terminologie plus «systématique», moins ponctuelle, permettrait de retenir des principes de rédaction plus stricts et observés de manière plus régulière, donnant la préférence au modèle de tel ou tel dictionnaire, ou décidant, en cas de définition multiple, de privilégier des systèmes de double ou triple définition etc. Le linguiste terminologue lexicologue aurait alors des lignes de conduite, mais qui seraient certainement de qualité conceptuelle différente de celle du spécialiste, seule à retenir de toutes façons dans le cas qui nous occupe.

7 Si ce Vocabulaire ne compte pas à sa nomenclature les termes « discours» ni « énoncé », on y trouve bien « variante », ou «contexte langagier», ce qui montre l'importance reconnue en terminologie de ces notions.
} 
simplement pour faire passer encore un peu de sa science vers son public de non spécialistes : «Le nom « hydrogène » désigne le mélange à l'état naturel des isotopes $1 \mathrm{H}, 2 \mathrm{H}$ et $3 \mathrm{H}$; il est également utilisé lorsqu'il n’est pas nécessaire de distinguer les divers isotopes " (sous protium). Bien des notes sont très clairement de nature encyclopédique. Ainsi, pour flux de travaux (pour l'angl. workflow) : «Un flux fait généralement l'objet de traitements par programmes informatiques ». Ou pour tautomérie de valence : «Par exemple, le bicyclopentène est un tautomère de valence du cyclopenta1,3-diène ». Ou encore sous byperconjugaison: « Le cation tertiobutyle et le toluène sont des exemples d'entités moléculaires pour lesquelles on admet une telle hyperconjugaison ».

On est amené à se demander si, chez les praticiens de la terminologie, le terme est considéré en tant que relevant d'un strict système notionnel, indépendamment du discours, ou si on reconnaitt au contraire au terme étudié un lien à la chaîne discursive, à ce discours qui l'actualise. Le lien est-il digne d'être mis en valeur ? Qu'en est-il du discours en terminologie?

\section{Le rôle avéré du « discours » en terminologie institutionnelle ${ }^{8}$}

\section{Le recours aux textes}

Quelle que soit « la diversité des textes qui peuvent être qualifiés de 'spécialisés' (...), « ces discours offrent un champ favorable au linguiste pour dégager les mécanismes sur lesquels repose la double fonction de la langue, de signifier et communiquer à la fois » (Mortureux 1997a : 185).

Une part importante de l'activité terminologique réside dans la recherche de paradigmes définitionnels ou désignationnels tels que les fait valoir M.-F. Mortureux (1993, 1997a et 1997b). A cet effet, la synthèse d'une riche documentation analytique mise à la disposition de l'ensemble des membres des commissions spécialisées et de la Commission générale de terminologie et de néologie depuis plusieurs années ${ }^{9}$ sert de support aux travaux.

L'expérience de la lexicographie générale a amplement démontré l'extrême utilité d'un vaste corpus textuel pour le traitement de mots "de la langue générale », voire pour celui de vocables «spécialisés ${ }^{10}$. De la même manière, l'expérience de la terminologie elle-même montre que le recours à de vastes ensembles d'attestations textuelles préexistantes est d'une valeur incomparable ${ }^{11}$. Qu'il nous soit permis d'insister, pour avoir été témoin d'une double expérience lexicographique (avec la

\footnotetext{
${ }^{8}$ Circuit de la terminologie institutionnelle, en France. Nous y participons en tant qu'expert linguiste, pour plusieurs commissions de terminologie et de néologie ; mais aussi, depuis novembre 1998 et par le biais de conventions périodiques entre la DGLFLF - Ministère de la Culture et notre laboratoire CNRS - Université Paris 7, pour l'ensemble des commissions de terminologie et de néologie.

${ }^{9}$ En fonction des engagements liant notre laboratoire et la DGLFLF, v. note 3.

${ }^{10}$ Nous pensons en particulier à la rédaction du Trésor de la langue française, expérience à laquelle nous avons eu l'occasion de faire référence ailleurs. Un article portant sur la néologie spécialisée dans le TLF est sous presse, à paraître chez Champion.

${ }^{11}$ Du moins telle qu'évoquée ici, dans le circuit du dispositif d'enrichissement de la langue française, terminologie ponctuelle multipôle.
} 
rédaction du Trésor de la langue française) et terminologique (avec l'expertise pour les Commissions de terminologie) ${ }^{12}$, sur l'utilité d'un tel recours à la source textuelle, même en terminologie. Cette source doit naturellement être sélectionnée, attestée et référencée de la manière la plus précise, préalable nécessaire à une bonne évaluation et une réutilisation adéquate.

Nous avons donc largement recours au discours. Avec les experts des domaines, nous utilisons et réutilisons les sources textuelles. Ces contextes sont de nature et d'origines diverses. Il s'agit le plus souvent, maintenant, de contextes trouvés sur l'Internet, source qui permet d'atteindre une large variété de discours, variété relative aux types de textes ou de sujets traités, aux degrés de spécialisation ou aux niveaux de langue. Ces paramètres d'approche lexicale, terminologique ou phraséologique, sont pris en compte, certes dans des proportions plus ou moins importantes.

Quoi qu'il en soit, et pour nous résumer, notons que le souci philologique, l'attachement aux textes perdure, en terminologie comme en lexicographie générale.

\section{Quels textes, et pour qui ?}

Les deux expériences citées, celle du TLF et celle des Commissions de terminologie, sont fort éloignées l'une de l'autre. Un point commun entre elles est sans doute d'avoir adopté, l'une comme l'autre, des frontières larges, ouvertes et souvent mal définies... Quel est vraiment le public ciblé ? Quelles sont les sphères d'activité dont on souhaite effectivement décrire le vocabulaire? Qui veut-on vraiment renseigner, et à quel sujet? Dans la pratique, on se pose ces questions de façon récurrente et la réponse est souvent impossible à donner. Il est vrai aussi que cet état de fait, observé d'abord de l'intérieur, par les praticiens de la terminologie et les premiers témoins, et qui apparaît comme un manque de rigueur ou de cohérence, se transforme aussi, finalement, en avantage : on peut faire ce constat au sujet du TLF aujourd'hui, et on le fera sans doute plus tard au sujet des listes de terminologie officielles. Moins se restreindre, c'est être moins clair dans ses objectifs; mais c'est aussi renseigner le plus grand nombre. Faire la part des choses entre les termes que l'on choisit de retenir, et, d'une certaine manière, de vulgariser, sélectionner les éléments sémantiques à mettre en avant dans les définitions, toutes ces activités impliquent, en amont, d'avoir à sa portée un vaste corpus d'usages réalisés et disponibles.

La richesse des textes mis à la disposition des Commissions spécialisées et de la Commission générale et la variété des experts qui y participent pourraient conférer à cette pratique terminologique des éléments favorables à une évaluation, une exploitation ou une orientation de nature socioterminologique, au sein desquelles pourraient être prises en compte les motivations des propositions ou des réactions individuelles en fonction des groupes professionnels, de leur degré de spécialisation, de leur domaine d 'expérience.

Ce corpus est celui des experts à travers leurs textes. Mais c'est aussi le discours des experts en direct, à travers leur présence aux commissions de terminologie et de

\footnotetext{
12 Expérience institutionnelle dans les deux cas : le dictionnaire du CNRS dans le premier cas, le dispositif d'enrichissement de la langue française dans le second.
} 
néologie, et leur participation, conditions sans lesquelles les commissions n'existeraient pas.

\section{Mais quelles recommandations officielles?}

Les différents pôles étudiés jusqu'ici pour situer la pratique terminologique sont représentés par les experts et leur science d'une part, les discours et les usages attestés, de l'autre. Les uns et les autres se rejoignent partiellement et diversement, concourant à un projet commun, le développement terminologique. Celui-ci ne peut cependant se réaliser sans une certaine médiatisation. La dernière étape est mise en scène, actualisée par un médiateur, qui peut être un reformulateur, ce «troisième homme» (Jacobi 1982 : 82) qui rend accessible une certaine forme de reformulation terminologique.

Mais cette dernière griffe du décideur est aussi, souvent, celle du généraliste. Les deux sont mêlées. Ce qui revient à dire que l'on surajoute une compétence de généraliste normalisateur à celle du spécialiste. De ce fait la norme n'est pas seulement représentée par les deux pôles mis en avant dans cette étude, la science et ses discours. Il faut ne pas sous-estimer une tierce force, celle du «décideur», officiel. C'est la norme de compétence du décideur.

Nous avons souligné quelques exemples de son influence : renvoyons une nouvelle fois aux recommandations officielles données pour "effet 'pogo' 》 (Sciences et techniques spatiales) et pour " raccourci au clavier» (Informatique) (Candel 2002 et 2003). Les formes bien en usage ${ }^{13}$ «effet pogo » et «raccourci-clavier», qui étaient bien celles que souhaitaient officialiser les deux commissions intéressées, ont été supplantées par ces autres, par souci de « correction».

Comme nous le rappelions au début, M.-F. Mortureux précise que, dans son titre La lexicologie entre langue et discours, "entre» exprime non un vide 'entre-deuxchaises', mais un va-et-vient» (p. 6). C'est aussi ce que nous avons tenté de démontrer au sujet de notre formule « la terminologie entre science et discours».

Mais lorsque les résultats s'éloignent des choix initiaux des commissions, comme nous venons de le montrer (avec « effet 'pogo' » par rapport à « effet pogo » et «raccourci au clavier» par rapport à «raccourci-clavier»), que reste-t-il de tout ce chemin laborieusement suivi au cœur des pôles «science » et « discours » ? Lorsque les décisions finalement s'écartent de ce qui avait été initialement prévu par les membres des commissions, il nous faut avouer que nous ne savons plus où situer les termes recommandés, ils se placent en tout cas en dehors de cette zone consensuelle recherchée entre les pôles "expertise scientifique » et "discours », et donc "entredeux-chaises » en effet.

L'intéressant, en vérité, est de savoir quand et pourquoi cette force de correction normalisatrice que nous avons symbolisée par le «tiers médiateur» intervient le plus volontiers. Sans doute est-ce lorsque le terme est relativement vulgarisé et que l'expert non spécialiste semble le comprendre : soit le terme lui est alors familier, en apparence su moins, du fait de sa forme; soit le terme a acquis pour

\footnotetext{
${ }^{13}$ Avant comme après l'officialisation des recommandations en question.
} 
lui une certaine limpidité, du fait d'indications supplémentaires fournies par les experts, et par le dossier de documentation proposé.

\section{Conclusion}

On a donc vu dans la première partie de cette étude que, selon certains chercheurs, terminologie et discours ne font pas bon ménage. Le rôle des experts est central lors des travaux pour les commissions de terminologie, comme on l'a vu dans la deuxième partie. La référence, le point de convergence, de stabilité, est à chercher, en ce sens, non seulement du côté de la langue, du système linguistique, mais aussi du côté de la science, des sciences. Le pôle «science » détermine l'origine et constitue, en même temps, le support de référence ; il cristallise le consensus culturel, scientifique, technique, professionnel des locuteurs. Dans la troisième partie, on a vu aussi à quel point la terminologie institutionnelle tient compte des contextes discursifs et de leurs réalités. On est amené à rappeler que le discours spécialisé, tel qu'on peut le collecter notamment à travers le vaste corpus de l'internet, est également disponible à la source, chez le spécialiste lui-même, dans l'expression de ses propres usages terminologiques et langagiers. M.-F. Mortureux cherche à «montrer l'interaction entre les contraintes linguistiques et les contraintes discursives dans le fonctionnement des mots » (1997b : 6). Montrer l'interaction entre les contraintes d'expertise scientifique et les contraintes discursives dans le développement terminologique est un autre programme, et auquel se surajoutent d'autres contraintes, plus directement normatives celles-là : rappelons en effet qu'il s'agit d'un dispositif d'enrichissement de la langue française officiel. 


\section{BIBLIOGRAPHIE}

Academic Press Dictionary for science and technology (1992), Academic Press.

Aussenac-Gilles N. et A. Condamines (éds) (2003), Rapport de l'Action Spécifique STIC «Corpus et Terminologie» (AS 34), Rattachée au RTP-DOC (RTP 33), Janvier 2002 juillet 2003, IRIT/2003-23-R, 80 p.

Boutin-Quesnel, R, N. BeLAnger, N. KerPan, L.-J. Rousseau (1985), Vocabulaire systématique de la terminologie, OLF, Québec, 38 p.

CABré, M. T. (1998 (1992)), La terminologie, Théorie, méthode et applications, Traduction, adaptation et mise à jour M. Cormier et J. Humbley, Les Presses de l'Université d'Ottawa, Armand Colin, 322 p.

CANDEL, D. (1999), «Néologie et terminologie : activités et réflexions », dans « Nouveaux outils de la néologie », in L.-J. Rousseau et L. Depecker (éds.), Terminologies nouvelles 20, p. 44-53.

CANDEL, D. (2002), «Images et usages de la norme : une observation des pratiques terminologiques au sein des Commissions de terminologie en France», in P. Bouchard et M. Cormier (éds), La représentation de la norme dans les pratiques terminologiques et lexicographiques, Office de la langue française, Québec, p. 93-105.

CANDEL, D. (2003), «Le processus officiel d'enrichissement de la langue française : quelques principes théoriques, leur histoire, leurs résultats pratiques ", in T. Slama-Cazacu (éd.), La langue et les parlants, Language and its users, Limba si vorbitorii, Arvin Press, Bucarest, p. 176-192.

Candel, D. et V. Toledano (2001), "Terminology on the move : selecting terms, writing definitions", 52 $2^{\text {nd }}$ International Astronautical Congress, 1-5 Octobre 2001, Toulouse, IAA paper IAA-01-IAA.5.1.07, p. 1-12.

Dubois, J., M. Giacomo, L. Guespin, C. Marcellesi, J.-B. Marcellesi, J.-P. Mevel (1973), Dictionnaire de linguistique, Larousse.

Dubois, J., M. Giacomo, L. Guespin, C. Marcellesi, J.-B. Marcellesi, J.-P. Mevel (1999 (1994)), Dictionnaire de linguistique et des sciences du langage, Larousse.

Gourseau, A., (1998), Dictionnaire de l'aéronautique et de l'espace, français-anglais, Gourseau.

Humbley, J. (2001), « Quelques enjeux de la dénomination en terminologie », in « Linguistique de la dénomination », Cahiers de praxématique 36, p. 117-139.

Humbley, J. (2004), «La réception de l'œuvre d'Eugen Wüster dans les pays de langue française ", in C. Cortès (éd.) "Terminologie : problèmes théoriques », Cahiers du CIEL 2004, Université Paris 7, p. 33-52.

JACOBI, D. (1982), «La diffusion des connaissances scientifiques - Quand des chercheurs diffusent les résultats de recherches du domaine des sciences de la vie dans une revue de vulgarisation », Les Cahiers du CRELEF 14, Université de Besançon, p. 81-96. 


\section{Danielle Candel}

JACOBI, D. (1987), Textes et images de la vulgarisation scientifique, Coll. Exploration Recherche en sciences de l'éducation, Peter Lang, 170 p.

JACOBI, D. (1997), «Petite revue des usages du lexique scientifique dans un texte de vulgarisation», Mélanges de linguistique offerts à Rostislav Kocourek, Les presses d'Alpha, Université Dalhousie, Halifax, Canada, p. 163-169.

Kucera, A., A. Clas, J. Baudot (1996), Dictionnaire compact des Sciences et de la Technique, Vol. I, Français - Allemand, Brandstetter Verlag, Wiesbaden.

Le grand dictionnaire terminologique, Office québécois de la langue française, Québec (Base de données en ligne, http://www.olf.gouv.qc.ca/ressources/gdt bdl2.html, interrogation de 1999).

Mortureux, M.-F. (1993), «Paradigmes désignationnels », Semen 8, Université de FrancheComté, CNRS, p. 121-141.

Mortureux, M.-F. (1994), «L'analyse du discours de la vulgarisation scientifique et le dictionnaire de la langue scientifique », dans «Français scientifique et technique et dictionnaire de langue », Etudes de sémantique lexicale, CNRS, Didier Erudition, p. 63-75.

Mortureux, M.-F. (1997a), «Synonymie et co-référence dans les discours spécialisés », Mélanges de linguistique offerts à Rostislav Kocourek, Les presses d'Alpha, Université Dalhousie, Halifax, Canada, p. 185-194.

Mortureux, M.-F. (1997b), « La lexicologie entre langue et discours », Coll. Campus, SEDES, $192 \mathrm{p}$.

Mots de la combustion (1993), Groupement français de combustion, section française du Combustion Institute [interrogation en ligne, 1999].

Pearson, J. (1998), Terms in context, John Benjamins, 242 p.

Termium, Bureau de la traduction, Ottawa, base de données terminologiques [interrogation en ligne, 1999].

Trésor de la langue française (1971-1994) (TLF), Dictionnaire de la langue française du 19e et du 20e siècle, CNRS, Klincksieck, puis Gallimard.

Williamson, M. (1990), Dictionary of Space Technology, Adam Hilger, Bristol and New York. 